ARTICLE

\title{
Effects of Human Model Configuration in Monte Carlo Calculations on Organ Doses from CT Examinations
}

\author{
Fumiaki TAKAHASHI ${ }^{1, *}$, Kaoru SATO ${ }^{1}$, Akira ENDO ${ }^{1}$, Koji ONO $^{2}$, Takayasu YOSHITAKE ${ }^{3}$, \\ Takayuki HASEGAWA $^{4}$, Yasushi KATSUNUMA ${ }^{5}$, Nobuhiko BAN ${ }^{6}$ and Michiaki KAI ${ }^{6}$ \\ ${ }^{1}$ Japan Atomic Energy Agency, 2-4 Shirakata, Tokai-mura, Ibaraki, 319-1195, Japan \\ ${ }^{2}$ Oita Prefecture-Mie Hospital, 3964-1 Miemachi-Miyano, Bungoono-shi, Oita, 879-7101, Japan \\ ${ }^{3}$ Shin-Beppu Hospital, 3898 Tsurumi, Beppu-shi, Oita, 874-0833, Japan \\ ${ }^{4}$ Tokai University Oiso Hospital, 21-1 Gakkyou, Oiso-machi, Kanagawa, 259-0198, Japan \\ ${ }^{5}$ Tokai University Hospital, 143 Shimokasuya, Isehara-shi, Kanagawa, 259-1193, Japan \\ ${ }^{6}$ Oita University of Nursing and Health Sciences, 2944-9 Megusuno, Oita-shi, Oita, 870-1201, Japan
}

\begin{abstract}
A new dosimetry system, WAZA-ARI, is being developed to estimate the radiation dose in Computed Tomography (CT) examinations in Japan. Dose estimation in WAZA-ARI utilizes organ dose data that are derived by Monte Carlo calculations using the Particle and Heavy Ion Transport code System (PHITS). A Japanese adult male phantom, JM phantom, has been adapted as a reference human model in the calculations, because the physique and inner organ masses agree well with the average values for Japanese adult males. However, since each patient has individual physical characteristics, the effect of a patient's body configuration on organ doses was studied by utilizing PHITS with another Japanese male model and the International Commission on Radiological Protection (ICRP) reference phantom. In addition, this paper describes computation conditions for the three human models, which were constructed in the form of voxel phantoms with different resolutions. Differences were observable among the phantoms in the dependences of the organ doses per air kerma on the position of the x-ray source running over the patient's body. The source model definition, however, more strongly affected the patient doses in the same phantom.
\end{abstract}

KEYWORDS: CT examination, organ doses, Monte Carlo calculation, PHITS code, voxel phantom, Japanese phantoms, ICRP reference phantom (AM phantom)

\section{Introduction}

Computed Tomography (CT) is currently advantageous in medical diagnosis. Here, it has been cautioned that a patient can receive a higher dose compared to other conventional radiography methods. ${ }^{1,2)}$ X-rays from CT examinations have become a major radiological source in many countries. ${ }^{1)}$ In particular, the number of CT examinations per capita in Japan is very large in the world. Thus, it is necessary to control the patient dose in CT examinations, while the radiation dose delivered to the patient should be sufficient for diagnostic imaging.

In this context, the new web-based system WAZA-ARI is being developed to achieve optimization of radiation exposure in CT examinations. ${ }^{3-5)}$ A user can set information on CT device conditions and scanning ranges. According to the given resources, WAZA-ARI can provide a radiation dose for a patient utilizing organ dose datasets, which have been prepared by Monte Carlo calculations using the Particle and Heavy Ion Transport code System (PHITS). ${ }^{6}$ A voxel-type phantom, the JM phantom, ${ }^{7)}$ is incorporated into PHITS in the numerical analyses. The physique and organs masses in the JM phantom are near to the average values for Japanese adult males. ${ }^{8)}$

It is important to study the effects of different human

*Corresponding author, E-mail:takahashi.fumiaki@jaea.go.jp

(C) 2011 Atomic Energy Society of Japan, All Rights Reserved. body configurations on the radiation dose, because a patient with an individual physique can be exposed to x-rays in a CT examination. Organ doses were therefore calculated using PHITS with two other voxel phantoms. One of these was another Japanese adult male phantom, named the Otoko phantom. ${ }^{9)}$ Although the height and weight agree well between the JM and Otoko phantoms, the inner configurations of the two phantoms are different. The other model was the International Commission on Radiological Protection (ICRP) reference Adult Male phantom (AM phantom). ${ }^{10)}$ The AM phantom, which was constructed from medical images of a Caucasian, has a larger physique than the JM and Otoko phantoms. Computation conditions were also compared among the Monte Carlo calculations using the three voxel phantoms.

\section{Monte Carlo Calculation}

\section{Human Models (Voxel Phantoms)}

Table 1 summarizes the main characteristics of the three voxel phantoms. The JM phantom was modified by removing the arms to calculate organ doses for the body (large) filter scanning option. ${ }^{4)}$ Analyses for the head (small) filter option adapt the original JM phantom with arms. However, the main characteristics are common between the two JM phantoms. 
Table 1 Main characteristics of the three phantoms

\begin{tabular}{cccc}
\hline & $\mathrm{JM}^{\mathrm{a}}$ & Otoko & $\mathrm{AM}^{10)}$ \\
\hline Subject & Japanese & Japanese & Caucasian \\
Height $(\mathrm{cm})$ & 171 & 170 & 176 \\
Weight $(\mathrm{kg})$ & 65 & 65 & 73 \\
\hline Resolution $(\mathrm{mm})$ & & & \\
Horizontal plane & $0.98 \times 0.98$ & $0.98 \times 0.98$ & $2.137 \times 2.137$ \\
Height & 1.0 & 10.0 & 8.0 \\
\hline Organ segmentation & ICRP & ICRP & ICRP \\
reference publication & Publ. 103 & Publ. 60 & Publ. 103 \\
\hline
\end{tabular}

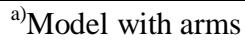

The Otoko phantom was constructed in the Japan Atomic Energy Agency (JAEA) from medical images of a Japanese subject with a height of $170 \mathrm{~cm}$ and weighting $65 \mathrm{~kg}$. Identification (ID) numbers corresponding to organs or tissues were assigned to all pixels in each image with a resolution of $0.98 \times 0.98 \mathrm{~mm}^{2}$ for the JM and Otoko phantoms. However, there is a tenfold difference in the image slice thickness, which is also the height resolution, between the JM and Otoko phantoms. The AM phantom was constructed on the basis of the Golem phantom, which was developed in the German Research Center for Environmental Health. ${ }^{10)}$ The physique and the masses of organs or tissues are adjusted to the basic anatomical and physiological data in ICRP Publication 89. ${ }^{11)}$ The resolution of the AM phantom is $2.137 \times 2.137 \mathrm{~mm}^{2}$ (horizontal plane) $\times 8.0 \mathrm{~mm}$ (height).

The organs and tissues, to which tissue weighting factors, $w_{T}$, are allocated in ICRP Publication 103, ${ }^{12)}$ are segmented in the JM and AM phantoms. Only the Otoko phantom was developed according to the concept of radiological protection dosimetry in ICRP Publication $60 .{ }^{13)}$

Table 2 Main characteristics of the three phantoms

\begin{tabular}{cccc}
\hline & JM with arms & Otoko & $\mathrm{AM}^{10)}$ \\
\hline Number of regions & 140 & 67 & 48 \\
Number of materials & 7 & 17 & 25 \\
Bone part (anatomy) & 20 & 17 & 19 \\
\hline Total mass (g) & 11,052 & 11,456 & 10,450 \\
Bone marrow (g) & 3,732 & 3,677 & 3,650 \\
\hline
\end{tabular}

Among the body tissues, the skeletal system consists of several materials with different elemental compositions. The atomic number of a material strongly affects energy deposition to the tissue in exposure from photons with energy below $150 \mathrm{keV}$. Thus, materials should be appropriately defined in each part of the anatomy from the cranium to the bones in the feet. Table 2 summarizes the definitions of the skeletal system in the three phantoms. The masses of bone tissues agree well with each other. The dose to the skeletal system was also derived from the masses in Table 2 for analyses using the JM phantom without arms. ${ }^{4}$ )

The JM phantom has 20 bone parts from an anatomical standpoint. Each part is divided into 7 regions with different materials based upon the thresholds of gray value reflecting density in each pixel. Thus, the JM phantom includes 140 $(=20 \times 7)$ regions for the skeletal system. ${ }^{7)}$ Table 3 lists the density and mass fractions of bone marrow (active and inactive) in each material. The material composition is presented in detail in Ref. 14). In the Otoko phantom, 67 bone regions can be anatomically grouped into 17 parts. The elemental composition was newly defined for the material in each part from the mass fraction of hard bone and bone marrow. The mass fraction of active bone marrow in each part to the whole skeletal system could be defined for the JM and Otoko phantoms from the data in ICRP Publication 89. ${ }^{11)}$ In the AM phantom, all of the bone parts consist of "cortical bone" and "spongiosa". ${ }^{10)}$ The latter material is specifically defined as the mixture of hard bone, cartilage and bone marrow in each bone part. In addition, "medullary" is defined for limb bones. ICRP Publication 110 presents the mass fractions of active bone marrow, inactive bone marrow and hard bone in the spongiosa for each part in the AM phantom.

Table 3 Skeletal system in the three male phantoms ${ }^{14)}$

\begin{tabular}{ccc}
\hline Material & Density $\left(\mathrm{g} \mathrm{cm}^{-3}\right)$ & Marrow (wt\%) \\
\hline 1 & 1.155 & 70.0 \\
2 & 1.244 & 54.0 \\
3 & 1.318 & 45.0 \\
4 & 1.388 & 36.0 \\
5 & 1.494 & 24.0 \\
6 & 1.641 & 10.0 \\
7 & 1.765 & 0.0 \\
\hline
\end{tabular}

\section{Application of Three Male Phantoms to PHITS}

The data for each phantom were saved in an ASCII format file, as listed in Table 4. The resolution in Table 1 closely relates to the total number of pixels. Outer pixels without body tissues are excluded from the JM phantom file because of the enormous data size. All of the phantom data were converted into the General Geometry (GG) format with the repeating structures using lattice descriptions, which are utilized to describe geometrical structures in the MCNP and MCNPX codes. $^{15,16)}$ In addition, using a newly introduced function, PHITS can read voxel phantom data in a binary format file. The radiation transport calculations in this study were made using the binary files of the voxel phantoms.

Table 4 Number of pixels and file size for the three phantoms

\begin{tabular}{cccc}
\hline Number of pixels & JM with arms $^{\text {a) }}$ & Otoko & AM $^{10)}$ \\
\hline Right-left & H 232, T 487, L 308 & 512 & 254 \\
Front-back & H 221, T 236, L 186 & 512 & 127 \\
Slice & H 260, T 840, L 735 & 179 & 222 \\
Total & $1.52 \times 10^{8}$ & $4.69 \times 10^{7}$ & $7.16 \times 10^{6}$ \\
\hline File size (ASCII) & $620 \mathrm{MB}$ & $217 \mathrm{MB}$ & $29 \mathrm{MB}$ \\
\hline
\end{tabular}

${ }^{\mathrm{a})} \mathrm{H}$ (Head), T (Torso) and L (Legs)

\section{Radiation Transport Calculations}

The "usrsors" subroutine in PHITS can define a source model, which describes the photon transport in a CT device, as depicted in Fig. 1. ${ }^{4)}$ The photon emission spot can be put at an arbitrary position on a circle surrounding the human model. The center of the circle corresponded to the center 
position of the images that were utilized to construct the JM and Otoko phantoms. The AM phantom was positioned in the same co-ordinate system. Configurations on the horizontal plane were mutually compared among the three phantoms at various heights (shoulders, chest, abdomen and legs). Photon emissions were into a fan-shaped region with a height of $5 \mathrm{~mm}$ corresponding to the energy distribution of $\mathrm{x}$-rays for each device. The photon flight direction was perpendicular to the long axis of the human model.

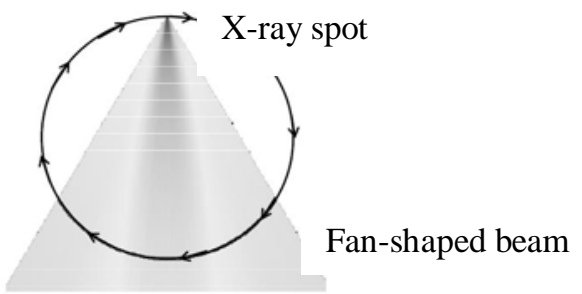

Fig. 1 The source model photon emissions (The spot is rotated on the circle in the dose calculation).

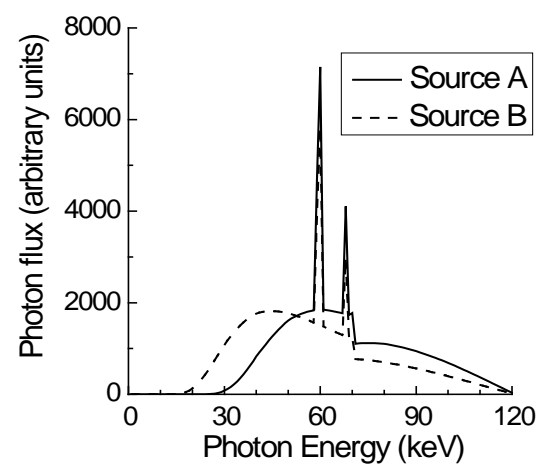

Fig. 2 Energy spectra of photons in the two source models.

All of the parameters are specifically defined in the source model for each CT device. WAZA-ARI already involves organ dose data corresponding to source (x-ray tube) positions on the human body for several 16-row multi-slice CT (16-row MSCT) devices with a body filter option and tube voltage of $120 \mathrm{kV}$. . The Otoko and AM phantoms were newly used in PHITS with the source models for two 16-row MSCT devices (hereinafter, Source A and Source B). Of the 16-row MSCT devices, the two source models were selected in this study, because the effects of the beam shaping (bow-tie) filter on photon attenuations in Source A and Source B were the smallest and the largest, respectively. Attenuation of photons in a beam-shaping filter can be numerically simulated by assigning a "weight" to each generated particle. As a beam-shaping filter stops more photon near the edge, the value of weight becomes a lower for photons emitted at larger angles. The photon energy is also a determining factor in defining the weight. Figure 2 depicts energy spectra in the two source models.

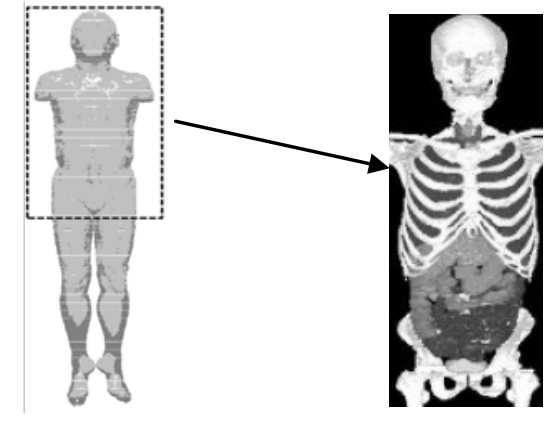

$\begin{array}{ll}\text { (a) Analysis range } & \text { (b) Inner configuration }\end{array}$

Fig. 3 Inner configuration of the JM phantom in the analyses range. ${ }^{7)}$

The source model movement was referred to previous analyses by the National Radiological Protection Board (NRPB, now the Health Protection Agency, HPA). ${ }^{17)}$ In organ dose calculations, the source model was moved in $5 \mathrm{~mm}$ steps from the vertex to the upper legs along the long axis of the body. Figure 3 shows the inner configuration of the JM phantom within the source movement range. Arm position should be taken into account for dose calculations. Photons emitted from the side of the body would be more shielded due to the arms in the Otoko and AM phantoms than in the armless JM phantom. Thus, numerical analyses were also performed using the JM phantom with arms for a number of source heights, although the calculated data are not utilized in WAZA-ARI.

In PHITS, the cut off energies of photons and electrons were set at $1 \mathrm{keV}$ and $10 \mathrm{keV}$ in the radiation transport, respectively. The organ doses could be obtained by dividing the deposited energies by the masses of organs and tissues. ${ }^{3}$ In addition, photon energy distributions were calculated at each bone part to correct differences in energy deposition between the active bone marrow and the material defined in the phantoms. As the JM and Otoko phantoms do not include cortical bone regions at the outermost parts of the bones, the radiation dose to the total bone was calculated as a replacement for the bone surface dose in this study.

Inner configurations of the body can influence the organ doses, because the patient is partially exposed to photons. ${ }^{3}$ ) The organ and tissue volumes were calculated for $5 \mathrm{~mm}$ step in the Otoko and AM phantoms within the analyzed range. The ray-tracing method using a plane source was applied to the volume calculations.

\section{Results and Discussion}

\section{Computation Time}

All of the radiation transport calculations were executed on a PC workstation, which was equipped with a $3.16 \mathrm{GHz}$ Intel Processor (E8500), 8.0 GB RAM and Scientific Linux 5.2 for x86_64. The computation time was an important issue in this study, because a large number of calculations were required to derive organ dose data for one source 
model. Table 5 lists computation times in the organ dose calculations with $1 \times 10^{6}$ generated photons. The source model was located on the abdomen, where the pelvis mass is the largest. At this height, the small and large intestines are involved. Most of statistical errors were within $1 \%$ for the energies deposited to these organs. (The maximum statistical error was less than $2 \%$.)

Table 5 Computation times for $1 \times 10^{6}$ particles (in minutes)

\begin{tabular}{cccc}
\hline Source , Averaged weight & JM & Otoko & AM \\
\hline Source A, 0.418 & 54 & 81 & 19 \\
Source B, 0.321 & 39 & 66 & 16 \\
\hline
\end{tabular}

Although the file size is the largest for the JM phantom, executing the organ dose calculation using the Otoko phantom took the longest time among the three phantoms. The JM phantom file included only the mesh regions without body tissues. Thus, it can be assumed that the computation time relates to the number of pixels on the horizontal plane rather than the total number of pixels. In addition to the human model, the source model affected the computation time.

\section{Inner Configurations of the Three Phantoms}

Figure 4 compares mass distribution of active bone marrow along the long axis of the body between the JM and AM phantoms. The data are plotted every $5 \mathrm{~mm}$ so as to correspond to the movement steps of the source model in the organ dose calculations. Throughout the rest of the paper, the height of the AM phantom is reduced by $5 \mathrm{~cm}$ to set the top of the head at $171 \mathrm{~cm}$, which corresponds to the height of the JM phantom. The data for the AM phantom would be shifted $5 \mathrm{~cm}$ higher, if the data are plotted at the "actual" height of this phantom. All organ dose data in the present paper are expressed using the values on the y-axis in Fig.4. The graph shows agreement in the mass distribution of active bone marrow between the two phantoms. The height around $90 \mathrm{~cm}$ includes the pelvis, in which the mass fraction of active bone marrow is large compared to other parts.

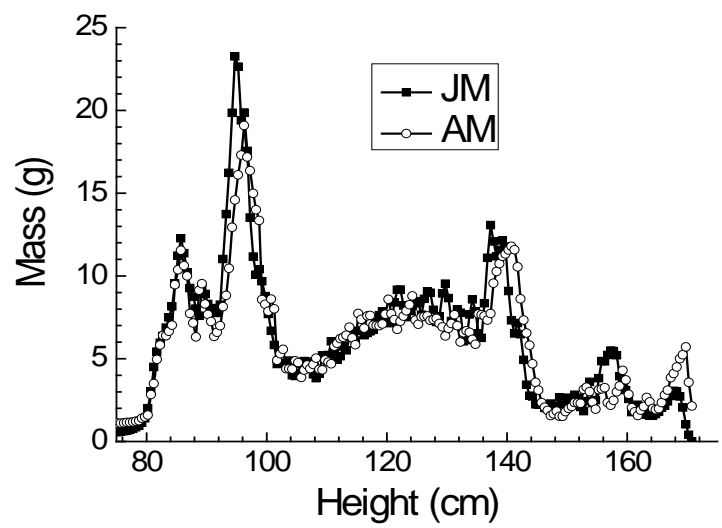

Fig. 4 Mass distribution of active bone marrow along the long axis of the body in the JM and AM phantoms.
Table 6 Organ configurations in the three phantoms

\begin{tabular}{cccc}
\hline Organs & JM & Otoko & $\mathrm{AM}^{10)}$ \\
\hline a) Range $(\mathrm{cm})^{\mathrm{a})}$ & & & \\
Thyroid & $140.0-145.0$ & $140.0-144.0$ & $141.0-146.5$ \\
Lungs & $114.5-142.0$ & $112.0-140.0$ & $120.5-142.5$ \\
Liver & $105.0-121.0$ & $104.0-121.0$ & $110.0-126.5$ \\
Stomach & $104.0-118.5$ & $112.0-118.0$ & $113.0-124.0$ \\
Colon & $84.0-112.5$ & $86.0-116.0$ & $86.0-121.0$ \\
Urinary bladder & $85.5-90.0$ & $86.0-90.0$ & $85.0-93.0$ \\
\hline b) Mass (g) & & & \\
Thyroid & 20.2 & 9.9 & 20.0 \\
Lungs & 1215 & 1358 & 1200 \\
Liver & 1462 & 1191 & 1800 \\
Stomach & 140.9 & 120.8 & 150.0 \\
Colon & 325.7 & 312.2 & 370.0 \\
Urinary bladder & 38.9 & 38.6 & 50.0 \\
\hline
\end{tabular}

a) The height on the y-axis in Fig. 4

Table 6 summarizes the ranges and masses of the thyroid, lungs, liver, stomach, colon and urinary bladder. The height of the Otoko phantom was increased by $1 \mathrm{~cm}$ here. Whereas the position of the thyroid is almost the same, most organs below the lungs are distributed at higher positions in the AM phantom than in the other two Japanese phantoms. The numerical data show that the bottom of the colon is not markedly different among the three models. This organ is supported by the pelvis. ${ }^{7)}$

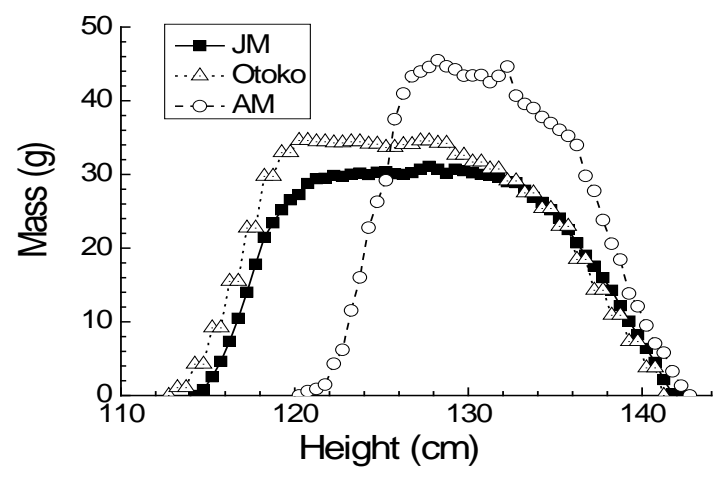

(a) Lungs

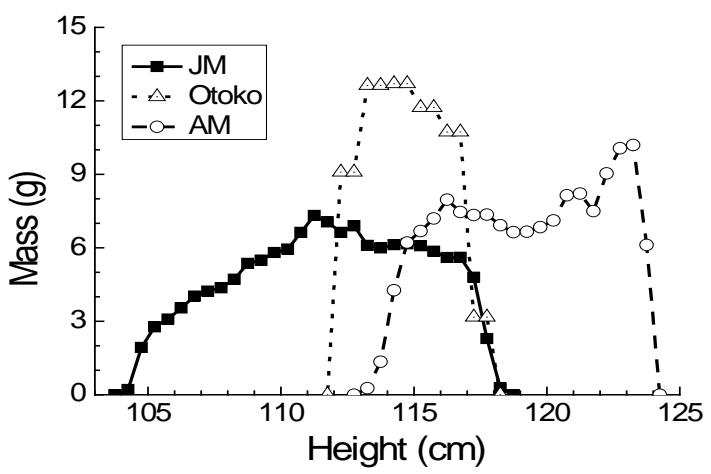

(b) Stomach

Fig. 5 Mass distribution of lungs and stomach along the long axis of the body in the three phantoms. 
Figure 5(a) shows that the base of the lungs differs between the AM phantom and the other two phantoms, although the apex of the lungs is almost the same among the three phantoms. The stomach position is higher in the AM phantom than the other two phantoms, as shown in Fig. 5(b). In addition, as the stomach configuration can easily change, ${ }^{7)}$ the length of the stomach along the long axis of the body is clearly different among the three phantoms, as also confirmed by the numerical values in Table 6 .

\section{Comparison of Organ Doses among the Three Phantoms}

WAZA-ARI datasets are prepared in the form of organ doses normalized to air kerma at the center of the photon emission points indicated in Fig. 1. ${ }^{4,5)}$ Figure 6 compares the data in WAZA-ARI with the normalized doses, which were derived from the radiation transport calculations using the Otoko and AM phantoms. The y-axis indicates the position of Source A along the long axis of the body.

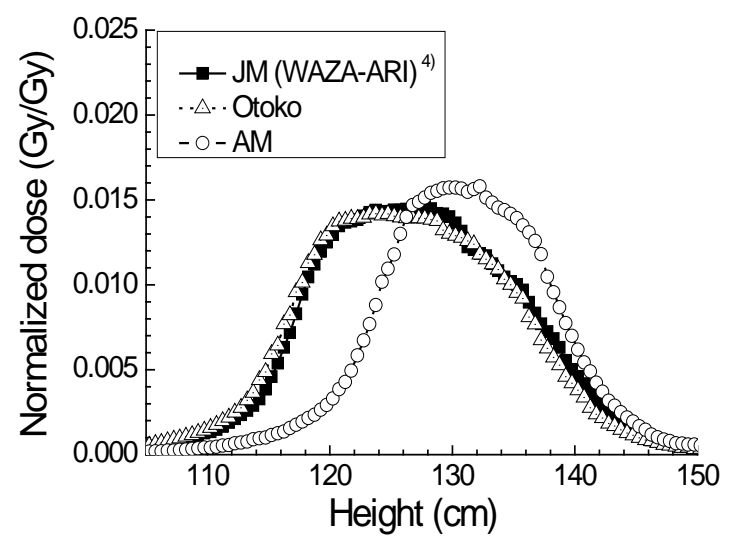

(a) Lungs

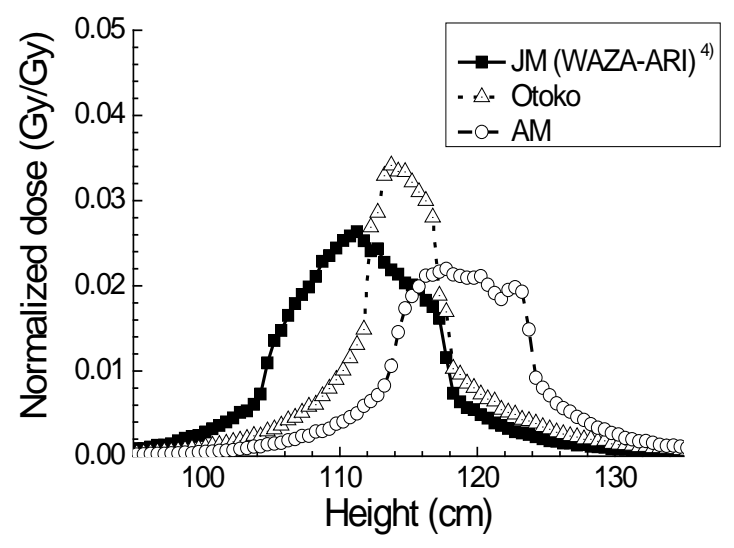

(b) Stomach

Fig. 6 Doses to lungs and stomach normalized to air kerma for the height of Source A.

The organ dose strongly depends on the mass fraction involved at the source height. ${ }^{3)}$ The normalized lung dose therefore decreases in the range between $120 \mathrm{~cm}$ and $130 \mathrm{~cm}$ in the AM phantom, while the doses are flat in the same range for the two Japanese adult male phantoms. The peak appears at different source heights for the normalized stomach dose in each phantom. The results clearly show that the scanning range can become an influential factor in organ dose calculations. WAZA-ARI can depict organs involved in a scanning range that is set by a user, with a display of the inner configuration of the JM phantom. ${ }^{5)}$

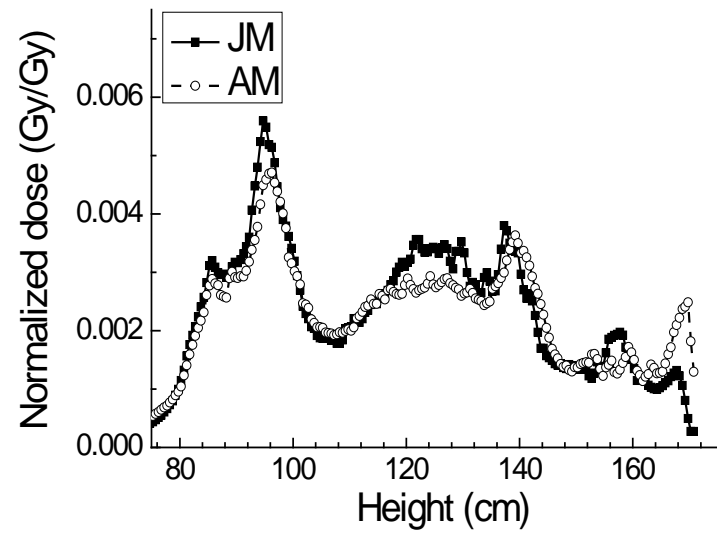

(a) Active bone marrow

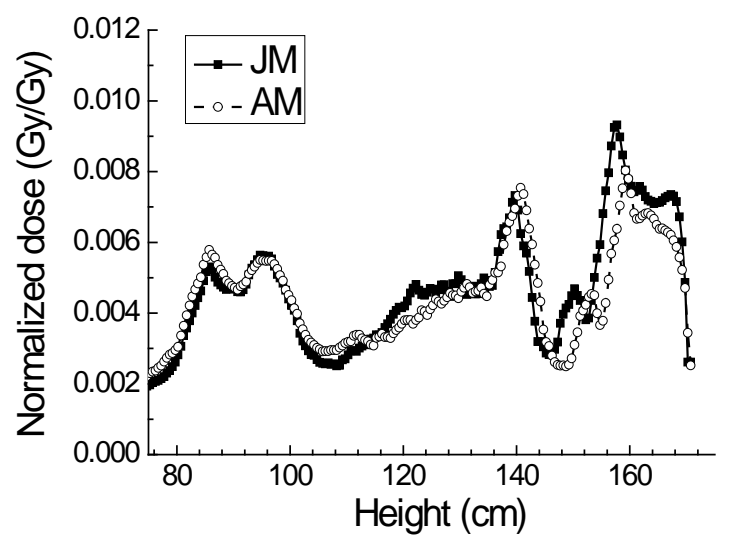

(b) Total bone

Fig. 7 Doses to active bone marrow and total bone normalized to air kerma for the height of Source A

Figure 7 shows normalized doses of active bone marrow and total bone for the movement of Source A on the JM and AM phantoms. Dependences of the normalized doses of active bone marrow and total bone on the source position are different due to the mass fraction along the long axis of the body. The total bone dose is large at the heights of the shoulders (around $140 \mathrm{~cm}$ ) and head (from $155 \mathrm{~cm}$ to $170 \mathrm{~cm}$ ). In addition to the large mass of bone, photons are not as strongly shielded by body tissues before reaching the bones in the shoulders and the head. A good agreement can be observed in the normalized dose of active bone marrow between the JM and AM phantoms. The materials and anatomical bone parts of the skeletal system are different 
between these two phantoms, as summarized in Table 2 . However, Figs. 4 and 7 indicate that the mass allocation and calculation of dose to active bone marrow are valid in numerical analyses using the JM phantom in the comparison with the ICRP reference AM phantom.

Table 7 Integrated normalized doses of some organs in the three phantoms (Gy/Gy)

\begin{tabular}{cccc}
\hline Source, Organs & JM without arms & Otoko & AM \\
\hline a) Source A & & & \\
Thyroid & 0.846 & 0.755 & 0.810 \\
Lungs & 0.624 & 0.618 & 0.538 \\
Liver & $0.620(0.577)^{\mathrm{a})}$ & 0.618 & 0.543 \\
Stomach & $0.663(0.632)$ & 0.572 & 0.553 \\
Colon & $0.649(0.616)$ & 0.611 & 0.588 \\
Urinary bladder & 0.592 & 0.547 & 0.601 \\
\hline b) Source B & & & \\
Thyroid & 0.658 & 0.587 & 0.634 \\
Lungs & 0.440 & 0.439 & 0.373 \\
Liver & $0.434(0.400)$ & 0.435 & 0.374 \\
Stomach & $0.473(0.448)$ & 0.391 & 0.383 \\
Colon & $0.463(0.439)$ & 0.435 & 0.417 \\
Urinary bladder & 0.410 & 0.372 & 0.416 \\
\hline
\end{tabular}

${ }^{\mathrm{a})}$ Calculated by the JM phantom with arms

The normalized doses were integrated over the range of source height along the long axis of the body, where the organ doses were more than $5 \%$ of the maximum value. Table 7 summarizes the integrated doses for the organs in Table 6. The unit of numerical values is of organ doses per air kerma (Gy/Gy).

Except for the stomach, the integrated doses agree within almost $10 \%$ between the Otoko phantom and the JM phantom without arms. The stomach distributions for the long axis (height) are $14.5 \mathrm{~cm}$ and $6 \mathrm{~cm}$ in the JM phantom and the Otoko phantom, respectively. Thus, the stomach can be exposed to photons within a shorter range of source model movement on the Otoko phantom.

The AM phantom provides the smallest integrated doses among the three phantoms, except the thyroid and the urinary bladder. The organ positions and body tissue thickness can affect the organ dose in the torso. The patient's posture is considered in the organ dose calculations used to prepare the datasets for the head and body filter options in WAZA-ARI. ${ }^{4)}$ The data show that the radiation dose to organs can change by about $5 \%$ with the existence of arms beside the torso, as confirmed with the doses in the two JM phantoms (with and without arms). The differences in the integrated doses are within about $15 \%$ between the AM phantom and the JM phantom in this case, where the position of the arms is set beside the torso.

All of the integrated doses are more strongly affected by the configuration of the source model. Integrated doses to all organs provided by Source A were about $40 \%$ larger than Source B. The user can select a CT device and a filter option in the dose calculation with WAZA-ARI. ${ }^{5}$

\section{Conclusions}

A web-based system, WAZA-ARI, is being developed to assess radiation doses to patients from CT examinations in Japan. The JM phantom has been adapted as a reference human model in WAZA-ARI. In the present study, two other human models, the Otoko and AM phantoms, were used in PHITS with the source models that can specifically simulate radiation transports in each CT device.

As confirmed previously, the organ doses changed according to the movement of a source model (x-ray tube) along the long axis of the body and thus differences could be observed in the relation between the source position and the organ doses due to the inner and outer configurations of the three voxel phantoms. The organ doses per air kerma were integrated over the range of interest of source model movement. The integrated dose to the stomach can differ by $20 \%$ among the three phantoms. On the other hand, the source model affected more strongly the integrated doses. The user can set the CT device and scanning condition in WAZA-ARI. In addition, the organs involved in the scanning range can be confirmed in WAZA-ARI by a display of JM phantom configuration and thus the uncertainty can be reduced in the dose calculation. This study also showed that the number of pixels on the horizontal plane can affect the dose calculation computation time using a voxel phantom.

\section{Acknowledgment}

The authors wish to thank GE Healthcare Japan, Hitachi Medical Corporation and Siemens Healthcare Japan (Siemens Asahi Medics Corporation) for their important help with our work.

This work has been supported by the Ministry of Education, Science, Sports and Culture (MEXT), Grant-inAid for Scientific Research (C), 20591484, 2008.

\section{References}

1) United Nations Scientific Committee on the Effects of Atomic Radiation, United Nations Scientific Committee on the Effects of Atomic Radiation 2000 Report to general assembly -Annex $D$ : Medical radiation exposures-, United Nations (UN) (2000).

2) IAEA, http://rpop.iaea.org

3) F. Takahashi, A. Endo, K. Sato, T. Hasegawa, Y. Katsunuma, K. Ono, T. Yoshitake, N. Ban, M. Kai, "Analysis of organ doses from Computed Tomography (CT) examination by the radiation transport calculation to develop the dosimetry system, WAZA-ARI,” Prog. Nucl. Sci. Technol., 1, 517-520 (2011).

4) F. Takahashi, A. Endo, K. Sato, K. Ono, T. Yoshitake, T. Hasegawa, Y. Katsunuma, N. Ban, M. Kai, "WAZA-ARI: Computational dosimetry system for x-ray CT examinations, I Radiation transport calculation for organ and tissue doses evaluation using JM phantom,” Radiat. Prot. Dosim., (in press).

5) N. Ban, F. Takahashi, K. Ono, T. Hasegawa, T. Yoshitake, Y. Katsunuma, K. Sato, A. Endo, M. Kai, "WAZA-ARI: 
Comupational dosimetry system for x-ray CT examinations, II Development of web-based system,” Radiat. Prot. Dosim., (in press).

6) H. Iwase, K. Niita, T. Nakamura, "Development of generalpurpose particle and heavy ion transport Monte Carlo code,” $J$. Nucl. Sci. Technol., 39 [11], 1142-1151 (2002).

7) K. Sato, F. Takahashi, D. Satoh, A. Endo, Development of adult voxel phantoms and their applications to evaluation of radiation exposure doses, JAEA-Data/Code 2010-009, Japan Atomic Energy Agency (JAEA) (2010).

8) G. Tanaka, H. Kawamura, Anatomical and physiological characteristics for Asian reference man -Male and female of different ages-, NIRS-M-115, National Institute on Radiological Sciences (NIRS) (1996).

9) K. Saito, A. Wittmann, S. Koga, Y. Ida, J. Kamei, Funabiki, "Construction of a computed tomographic phantom for a Japanese adult male and dose calculation system,” Radiat. Environ. Biophys., 40, 69-76 (2001).

10) International Commission on Radiological Protection, "Adult reference computational phantoms; ICRP Publication 110," Ann. ICRP, 39 (2009).

11) International Commission on Radiological Protection, "Basic anatomical and physiological data for use in radiological protection; ICRP Publication 89," Ann. ICRP, 32 (2002).
12) International Commission on Radiological Protection, "The 2007 recommendations of the International Commission on Radiological Protection; ICRP Publication 103," Ann. ICRP, 37 (2007).

13) International Commission on Radiological Protection, "The 1990 recommendations of the International Commission on Radiological Protection; ICRP Publication 60," Ann. ICRP, 21 (1991).

14) D. Satoh, K. Sato, F. Takahashi, A. Endo, "Monte Carlo simulation using Japanese voxel phantoms to analyze the contribution of particle types and their energy distributions to organ doses upon external neutron exposure," J. Nucl. Sci. Technol., 47[1], 62-69 (2010).

15) X-5 Monte Carlo Team, MCNP - A general Monte Carlo n- $p$ particle transport code version 5, LA-UR-03-1987, Los Alamos National Laboratory (LANL) (2003).

16) L. S. Waters (ed.), MCNPX user's manual version2.3, LA-UR-02-2607, Los Alamos National Laboratory (LANL) (2003).

17) D. G. Jones, P. C. Shrimpton, Survey of CT practice in the UK- Part III: Normalised organ doses calculated using Monte Carlo techniques-, NRPB-R250, National Radiological Protection Board (NRPB) (1991). 